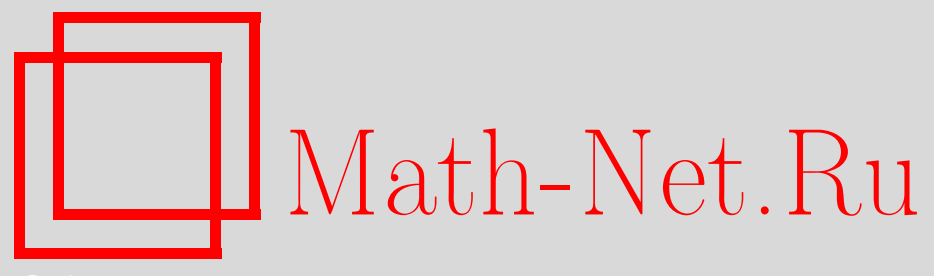

В. Е. Назайкинский, Геометрия фазового пространства для волнового уравнения, вырождающегося на границе области, Матем. заметки, 2012, том 92, выпуск 1, 153156

DOI: https://doi.org/10.4213/mzm9488

Использование Общероссийского математического портала Math-Net.Ru подразумевает, что вы прочитали и согласны с пользовательским соглашением http: //www . mathnet.ru/rus/agreement

Параметры загрузки:

IP: 54.89 .56 .158

26 апреля 2023 г., $16: 33: 30$

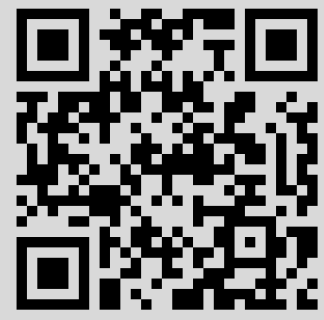




\section{Геометрия фазового пространства для волнового уравнения, вырождающегося на границе области}

\section{В. Е. Назайкинский}

1. Введение. Вырождающиеся на границе области волновые уравнения часто встречаются в приложениях. Например, они возникают при моделировании в линейном приближении наката на берег волн цунами [1], [2]. Асимптотику решений уравнений волнового типа естественно строить методом канонического оператора (см., например, [3]), который опирается на геометрию траекторий отвечающей рассматриваемому уравнению системы Гамильтона. В отличие от невырожденного случая (который для уравнений мелкой воды с локализованными начальными данными был исследован этим методом в [4], [5]; см. также цитированную там литературу), геометрия траекторий системы Гамильтона для вырождающегося уравнения имеет следующую особенность: они уходят на бесконечность по импульсам за конечное время, одновременно подходя к границе в проекции на координатное пространство. Поэтому для успешного решения задачи необходимо правильно описать, что происходит с траекториями после ухода на бесконечность (т.е. найти закон отражения от границы) и построить канонический оператор на возникающих при этом неограниченных лагранжевых многообразиях. В одномерном случае это было сделано в [6], и результаты были применены в [7] для построения асимптотик в одномерной задаче Коши для вырождающегося волнового уравнения с локализованными начальными данными. Модельная двумерная задача в полуплоскости с вырождением на граничной прямой была рассмотрена в [8].

В данной работе мы изучаем отвечающую вырождающимся волновым уравнениям геометрию фазового пространства для области общего вида и произвольной размерности. (Заметим, что предлагаемое геометрическое описание является новым и в одномерном случае.) Конструкция канонического оператора и формулы для асимптотических решений будут даны в отдельной статье. Доказательства всех результатов будут приведены в подробной публикации.

2. Вырожденное волновое уравнение и его гамильтониан. Пусть $M$ - ограниченная замкнутая область с гладкой границей $\partial M$ и внутренностью $\Omega=M \backslash \partial M$ в $n$-мерном пространстве $\mathbb{R}^{n}$ с координатами $x=\left(x_{1}, \ldots, x_{n}\right)$. Уравнение вида

$$
\frac{\partial^{2} u}{\partial t^{2}}-\sum_{j, k=1}^{n} \frac{\partial}{\partial x_{j}}\left(a_{j k}(x) \frac{\partial u(x)}{\partial x_{k}}\right)=0, \quad u=u(x, t), \quad x \in \Omega, \quad t \in[0, T],
$$

будем называть (обобщенным) волновым уравнением, вырождающимся на границе области $M$, если матрица его коэффициентов $A(x)=\left(a_{j k}(x)\right)$ представима в виде $A(x)=$ $\varphi(x) B(x)$, где $B(x)$ - симметрическая вещественная матрица, гладко зависящая от $x$ и строго положительно определенная при всех $x \in M$, а $\varphi(x)$ - определяющая функиия границы $\partial M$ (т.е. гладкая функция в $M$, положительная в $\Omega$ и равная нулю на $\partial M$, градиент которой не обращается в нуль на $\partial M)$. В частности, если $B(x)$ - единичная матрица, то уравнение (1) представляет собой волновое уравнение в $\Omega$ со скоростью $c(x)=\sqrt{\varphi(x)}$, вырождающейся на $\partial M$ как квадратный корень из расстояния до $\partial M$. Отвечающий уравнению (1) гамильтониан имеет вид

$$
H(x, p)=\langle p, A(x) p\rangle^{1 / 2}=\sqrt{\varphi(x)}\langle p, B(x) p\rangle^{1 / 2} .
$$

Работа выполнена при поддержке Российского фонда фундаментальных исследований (грант № 11-01-00973-a). 
(Здесь и далее $\langle\xi, \eta\rangle=\sum_{j=1}^{n} \xi_{j} \eta_{j}$.) Траектории соответствующей гамильтоновой системы

$$
\dot{x}=H_{p} \equiv A(x) p\langle p, A(x) p\rangle^{-1 / 2}, \quad \dot{p}=-H_{x} \equiv-\frac{1}{2}\left\langle p, \frac{\partial A}{\partial x}(x) p\right\rangle\langle p, A(x) p\rangle^{-1 / 2}
$$

в фазовом пространстве $T_{0}^{*} \Omega \simeq \Omega \times\left(\mathbb{R}^{n} \backslash\{0\}\right)$ не являются бесконечно продолжимыми: за конечное время они уходят на бесконечность по импульсным переменным $p$, при этом приближаясь по переменным $x$ к границе $\partial M$ области $M$.

3. Фазовое пространство. Построим "пополненное" фазовое пространство $\Phi \supset T_{0}^{*} \Omega$ таким образом, чтобы выполнялись следующие условия.

1. Исходное фазовое пространство $T_{0}^{*} \Omega$ - открытое подмножество в гладком многообразии $\Phi$, причем $\Phi \backslash T_{0}^{*} \Omega$ - гладкое подмногообразие размерности $2 n-1$.

2. Многообразие $\Phi-$ симплектическое с (гладкой) структурной формой $\omega^{2}$, причем вложение $T_{0}^{*} \Omega \subset \Phi$ является вложением симплектических многообразий, т.е.

$$
\left.\omega^{2}\right|_{T_{0}^{*} \Omega}=d p \wedge d x \equiv \sum_{j=1}^{n} d p_{j} \wedge d x_{j} .
$$

3. Гамильтониан $H(x, p)$ продолжается (по непрерывности) до гладкой функции на $\Phi$.

4. Как следствие, гамильтоново векторное поле (3) продолжается по непрерывности до гладкого векторного поля на $\Phi$, причем траектории последнего неограниченно продолжимы вперед и назад по времени.

Пусть $U \subset M$ - достаточно малая воротниковая окрестность в $M$ края $\partial M$ области $M$, $\psi(x)$ - некоторая положительная в $\Omega$ определяющая функция края $\partial M$, и пусть $a(x)-$ гладкое векторное поле в $U$ такое, что

$$
\left\langle a(x), \psi^{\prime}(x)\right\rangle=1 \quad \text { при } \quad x \in U ;
$$

например, $a(x)=b(x)\left\langle b(x), \psi^{\prime}(x)\right\rangle^{-1}$, где $b(x)$ - векторное поле, трансверсальное границе $\partial M$ (знаменатель не обращается в нуль, если окрестность $U$ достаточно мала).

В прямом произведении $T^{*} U \times \mathbb{R} \times(0, \infty)$ с координатами $(y, \eta, q, E),(y, \eta) \in T^{*} U, q \in \mathbb{R}$, $E \in(0, \infty)$, рассмотрим подмножество

$$
V=\left\{(y, \eta, q, E) \in T^{*} U \times \mathbb{R} \times(0, \infty):\langle a(y), \eta\rangle=0, \psi(y)=q^{2} E\right\}
$$

и его подмножество $V_{\infty}=\{(y, \eta, q, E) \in V: q=0\}$.

Лемма 1. Множество $V$ представляет собой гладкое $2 n$-мерное подмногообразие в $T^{*} U \times \mathbb{R} \times(0, \infty)$, a $V_{\infty}$ - гладкое $(2 n-1)$-мерное подмногообразие в $\mathrm{V}$.

$\mathrm{B}$ пространстве $T_{0}^{*} \Omega$ с координатами $(x, p)$ определим гладкое $(2 n-1)$-мерное подмногообразие

$$
W_{0}=\left\{(x, p) \in T_{0}^{*} \Omega: x \in U \backslash \partial M,\langle a(x), p\rangle=0\right\}
$$

и зададим отображение

$$
f: V \backslash V_{\infty} \rightarrow T_{0}^{*} U \backslash W_{0} \subset T_{0}^{*} \Omega
$$

формулами

$$
x=y, \quad p=\eta+\frac{\psi^{\prime}(x)}{q} .
$$

Лемма 2. Отображение (7), (8) корректно определено и является диффеоморфизмом. Обратное отображение $f^{-1}: T_{0}^{*} U \backslash W_{0} \rightarrow V \backslash V_{\infty}$ задается формулами

$$
y=x, \quad \eta=p-\langle a(x), p\rangle \psi^{\prime}(x), \quad q=\frac{1}{\langle a(x), p\rangle}, \quad E=\psi(x)\langle a(x), p\rangle^{2} .
$$


Положим теперь $\Phi=V \sqcup_{f} T_{0}^{*} \Omega$ (т.е. в дизъюнктном объединении $V \sqcup T_{0}^{*} \Omega$ отождествим точки, связанные диффеоморфизмом $f$ ) и снабдим это множество естественной топологий, базу которой образуют образы открытых множеств при естественных вложениях $V \subset \Phi$ и $T_{0}^{*} \Omega \subset \Phi$.

ПредложениЕ 1. Множество $\Phi$ наделено естественной структурой гладкого многообразия, при которой вложения $V \subset \Phi$ и $T_{0}^{*} \Omega \subset \Phi$ суть вложения гладких многообразий. Более того, $\Phi=T_{0}^{*} \Omega \sqcup V_{\infty}$, причем $T_{0}^{*} \Omega$ - открытое всюду плотное подмногообразие, а $V_{\infty}-(2 n-1)$-мерное подмногообразие.

Представленная выше конструкция пространства $\Phi$ содержит произвольно выбираемые окрестность $U$, функцию $\psi$ и векторное поле $a$. Однако само пространство $\Phi$ с точностью до диффеоморфизма не зависит от произвола в этой конструкции. Более точно, пусть $\Phi_{1}$ и $\Phi_{2}$ - два разных пространства $\Phi$, полученных из $T_{0}^{*} \Omega$ такой конструкцией.

Предложение 2. Тождественное отображение $\Phi_{1} \supset T_{0}^{*} \Omega \stackrel{\mathrm{id}}{\longrightarrow} T_{0}^{*} \Omega \subset \Phi_{2}$ продолжается по непрерывности до диффеоморфизма многообразия $\Phi_{1}$ на многообразие $\Phi_{2}$.

Указанная конструкция, будучи применена локально, дает удобные локальные координаты на $\Phi$ в окрестности произвольной точки “подмногообразия на бесконечности" $V_{\infty}$. Именно, возьмем произвольную точку границы $\partial M$. В окрестности этой точки определяющую функцию границы (при необходимости перенумеровывая координаты и меняя знак координаты $x_{1}$ ) можно записать в виде $\psi(x)=x_{1}-g\left(x^{\prime}\right)$, где $g\left(x^{\prime}\right)$ - гладкая функция переменных $x^{\prime}=\left(x_{2}, \ldots, x_{n}\right)$, а векторное поле взять равным $a(x)=(1,0, \ldots, 0)$. Тогда множество $V$ над такой окрестностью задается уравнениями

$$
y_{1}=g\left(y^{\prime}\right)+q^{2} E, \quad \eta_{1}=0,
$$

и в качестве локальных координат на $V$ (и, следовательно, на $\Phi)$ в окрестности соответствующих точек из $V_{\infty}$ можно взять $\left(q, y^{\prime}, E, \eta^{\prime}\right)$.

ПредложениЕ 3. Симплектическая форма $d p \wedge d x$ на $T_{0}^{*} \Omega$ продолжается до замкнутой невырожденной 2-формы $\omega^{2}$ на $\Phi$. В указанных выше локалъных координатах в окрестности точек из $V_{\infty}$ форма $\omega^{2}$ записывается в виде

$$
\omega^{2}=d E \wedge d q+d \eta^{\prime} \wedge d y .
$$

Итак, многообразие $\Phi$ обладает симплектической структурой, естественно продолженной с исходного фазового пространства, и для любой гладкой функции на $\Phi$ определена соответствующая система Гамильтона.

ПредложениЕ 4. Функиия Гамильтона (2) может быть продолжена по непрерывности до гладкой функиии $\widetilde{H}$ на $\Phi$, и траектории системы Гамильтона, соответствующей $\widetilde{H}$ (которые содержат в качестве подынтервалов все траектории системы Гамильтона (3)), неограниченно продолжсаются вперед и назад по времени.

Комбинируя предложения 1,3 и 4, приходим к следующему утверждению.

Теорема 1. Фазовое пространство $\Phi$ с формой $\omega^{2}$ удовлетворяет условиям 1-4.

4. Пример. Пусть $\Omega$ - внешность эллипса $x_{1}^{2} / a_{1}^{2}+x_{2}^{2} / a_{2}^{2}=1$, а гамильтониан имеет вид

$$
H(x, p)=\left(\frac{r-1}{r+1}\left(p_{1}^{2}+p_{2}^{2}\right)\right)^{1 / 2}, \quad \text { где } \quad r=\left(\frac{x_{1}^{2}}{a_{1}^{2}}+\frac{x_{2}^{2}}{a_{2}^{2}}\right)^{1 / 2} .
$$

В полярных координатах $(r, \varphi)$, задаваемых формулами $x_{1}=a_{1} r \cos \varphi, x_{2}=a_{2} r \sin \varphi$, гамильтониан записывается в виде

$$
H(r, \varphi, P, Q)=\left[\frac{r-1}{r+1}\left\langle\left(\begin{array}{l}
P \\
\frac{Q}{r}
\end{array}\right)\left(\begin{array}{cc}
\frac{\cos ^{2} \varphi}{a_{1}^{2}}+\frac{\sin ^{2} \varphi}{a_{2}^{2}} & \frac{\sin 2 \varphi}{2}\left(\frac{1}{a_{2}^{2}}-\frac{1}{a_{1}^{2}}\right) \\
\frac{\sin 2 \varphi}{2}\left(\frac{1}{a_{2}^{2}}-\frac{1}{a_{1}^{2}}\right) & \frac{\sin ^{2} \varphi}{a_{1}^{2}}+\frac{\cos ^{2} \varphi}{a_{2}^{2}}
\end{array}\right)\left(\begin{array}{l}
P \\
\frac{Q}{r}
\end{array}\right)\right]^{1 / 2},\right.
$$


где $P=a_{1} p_{1} \cos \varphi+a_{2} p_{2} \sin \varphi$ и $Q=r\left(a_{2} p_{2} \cos \varphi-a_{1} p_{1} \sin \varphi\right)-$ импульсы, двойственные к $r$ и $\varphi$, соответственно, так что $P d r+Q d \varphi=p_{1} d x_{1}+p_{2} d x_{2}$. В полярных координатах область $\Omega$ имеет вид $r>1$, поэтому в качестве координат на $\Phi$ введем следующие функции:

$$
q=\frac{1}{P}, \quad E=(r-1) P^{2}, \quad \varphi, \quad Q .
$$

Тогда, в соответствии с общей теорией,

$$
d P \wedge d r+d Q \wedge d \varphi=d E \wedge d q+d Q \wedge d \varphi,
$$

а гамильтониан является гладкой функцией новых координат, в том числе и при $q=0$ :

$$
\begin{aligned}
H & (q, \varphi, E, Q) \\
& =\left\{\frac{E}{E q^{2}+2}\left\langle\left(\begin{array}{c}
1 \\
\frac{q Q}{E q^{2}+1}
\end{array}\right)\left(\begin{array}{cc}
\frac{\cos ^{2} \varphi}{a_{1}^{2}}+\frac{\sin ^{2} \varphi}{a_{2}^{2}} & \frac{\sin 2 \varphi}{2}\left(\frac{1}{a_{2}^{2}}-\frac{1}{a_{1}^{2}}\right) \\
\frac{\sin 2 \varphi}{2}\left(\frac{1}{a_{2}^{2}}-\frac{1}{a_{1}^{2}}\right) & \frac{\sin ^{2} \varphi}{a_{1}^{2}}+\frac{\cos ^{2} \varphi}{a_{2}^{2}}
\end{array}\right)\left(\begin{array}{c}
1 \\
\frac{q Q}{E q^{2}+1}
\end{array}\right)\right\rangle\right\} \\
& =\left\{\frac{E}{E q^{2}+2}\left[\frac{\cos ^{2} \varphi}{a_{1}^{2}}+\frac{\sin ^{2} \varphi}{a_{2}^{2}}+\frac{q Q \sin 2 \varphi}{E q^{2}+1}\left(\frac{1}{a_{2}^{2}}-\frac{1}{a_{1}^{2}}\right)+\frac{q^{2} Q^{2}}{\left(E q^{2}+1\right)^{2}}\left(\frac{\sin ^{2} \varphi}{a_{1}^{2}}+\frac{\cos ^{2} \varphi}{a_{2}^{2}}\right)\right]\right\}^{1 / 2} .
\end{aligned}
$$

Из выражения (10) для симплектической формы следует, что система Гамильтона имеет стандартный вид

$$
\dot{q}=H_{E}, \quad \dot{E}=-H_{q}, \quad \dot{\varphi}=H_{Q}, \quad \dot{Q}=H_{\varphi} .
$$

Мы не будем явно выписывать соответствующие громоздкие выражения, а отметим лишь, что при $q=0$ справедливо соотношение

$$
\dot{q}=\frac{1}{4 H}\left[\frac{\cos ^{2} \varphi}{a_{1}^{2}}+\frac{\sin ^{2} \varphi}{a_{2}^{2}}\right] \neq 0 .
$$

Таким образом, траектории системы Гамильтона трансверсальны “подмногообразию на бесконечности" $V_{\infty}$ фазового пространства $\Phi$.

Автор признателен С. Ю. Доброхотову за внимание и поддержку.

\section{СПИСОК ЦИТИРОВАННОЙ ЛИТЕРАТУРЫ}

[1] J. J. Stoker, Water Waves. The Mathematical Theory with Applications, Pure Appl. Math., 4, Interscience Publ., New York, 1957. [2] Е. Н. Пелиновский, Гидродинамика волн иунами, ИПФ РАН, Нижний Новгород, 1996. [3] В. П. Маслов, М. В. Федорюк, Квазиклассическое приближение для уравнений квантовой механики, Наука, M., 1976. [4] S. Yu. Dobrokhotov, R. V. Nekrasov, B. Tirozzi, J. Engrg. Math., 69:2-3 (2011), 225-242. [5] С. Ю. Доброхотов, Б. Тироцци, УМH, 65:1 (2010), 185-186. [6] Т. Vukašinac, P. Zhevandrov, Russ. J. Math. Phys., 9:3 (2002), 371-381. [7] S. Yu. Dobrokhotov, V.E. Nazaikinskii, B. Tirozzi, Russ. J. Math. Phys., 17:4 (2010), 434-447. [8] С. Ю. Доброхотов, В. Е. Назайкинский, Б. Тироцци, Алгебра и анализ, 22:6 (2010), 67-90.

\section{В. Е. Назайкинский}

Московский государственный институт электроники и математики (технический университет)

E-mail: nazaikinskii@yandex.ru 